\title{
Preoperative High Neutrophil-to-Lymphocyte Ratio Is Associated with High-grade Bladder Cancer
}

\author{
XINGXING TANG*, SHUO WANG*, CHAO AN, PENG DU and YONG YANG \\ Key Laboratory of Carcinogenesis and Translational Research (Ministry of Education), \\ Department of Urology, Peking University Cancer Hospital \& Institute, Beijing, P.R. China
}

\begin{abstract}
Aim: To evaluate the correlation between the neutrophil-to-lymphocyte ratio (NLR) and histopathological characteristics of bladder cancer. Materials and Methods: A retrospective analysis was performed on a cohort of patients $(n=302)$ who were diagnosed with bladder cancer and underwent transurethral resection of bladder tumor or cystectomy between 2009 and 2016. The pathological outcomes were compared between patients with low NLR and those with high NLR with a cut-off value of 2.5 , and a logistic regression analysis was performed to find potential predictors of pathological tumor outcomes. Results: Patients with highgrade disease had significantly higher NLRs compared to those with low-grade disease (median $N L R=4.42 \mathrm{vs} .3 .42$, $p<0.001)$. Univariate analysis suggested that age, neutrophil count and NLR, as a continuous or binary variable, were significantly associated with high-grade disease. Multivariate analysis suggested that age and NLR, as a continuous variable, were predictors of pathologically high-grade disease. Conclusion: Preoperative NLR was found to be associated with pathological tumor grading, but was not associated with pathological tumor staging in patients with bladder cancer.
\end{abstract}

Based on recent theories, systemic inflammation induced by tumors might play a critical role in tumor occurrence, progression and metastasis (1). The neutrophil count rises while the lymphocyte count falls under systemic inflammation, which leads to a change in the neutrophil to lymphocyte ratio (NLR) (2). As a marker of systemic inflammation, the NLR has been evaluated as an effective

*These Authors contributed equally to the present study.

Correspondence to: Peng Du, Key Laboratory of Carcinogenesis and Translational Research (Ministry of Education), Department of Urology, Peking University Cancer Hospital \& Institute, No. 52 Fucheng Road, Haidian District, Beijing 100142, P.R. China. Email: dupeng9000@126.com

Key Words: Neutrophil to lymphocyte ratio, bladder cancer, pathological outcome. predictive marker for a range of cancer typess, including gastrointestinal (3) and epithelial ovarian (4).

Several studies have suggested that NLR may be a predictor of poor survival in patients with bladder cancer (58). However, the link between elevated NLR and poor survival remains controversial. Pathological differences between patients with a low NLR and those with a high NLR might be one important reason for this. In order to investigate associations between pathological differences and NLR, we conducted this study in patients with bladder cancer.

\section{Materials and Methods}

Study population. A retrospective analysis was performed to identify patients with bladder cancer at Peking University Cancer Hospital between 2009 and 2016. The inclusion criterion were: a) adult patients aged 18 years or older, male or female; b) diagnosed with bladder cancer for the first time before surgery; c) transurethral resection of the bladder tumor or radical cystectomy; d) bladder urothelial carcinoma was confirmed by pathology report with complete grading and staging information. The exclusion criterion were: a) other types of bladder cancer, such as squamous carcinoma, metastatic cancer; b) factors potentially influencing the NLR, such as infection and leukocytosis.

Data collection. The data for basic patient information, laboratory results and pathological results were collected from the electronic patient records. All hematological parameters were calculated prior to operations. The pathological staging and histological grading of the bladder cancer were based on the American Joint Committee TNM Staging System for Bladder Cancer (seventh edition) (9). Data obtained from the patients' routine tests before surgery included the white blood cell count (WBC), neutrophil count and lymphocyte count. The NLR was defined by dividing the neutrophil count by the lymphocyte count and the NLR cut-off value was 2.5 from previous publications, with an abnormal value defined as $>2.5$ (6).

Statistical analysis. Descriptive statistics were used to summarize patients' characteristics. Categorical variables are presented as numbers and percentages and continuous variables as median and interquartile range (IQR). Patients were divided into two groups by pathological tumor grading, low grade and high grade. The $t$-test was used to compare the continuous variables between patients with low-grade disease and those with high-grade disease. Chi-square test 
was used to compare the categorical variables between these two groups. Patients were then divided into two groups with a cut-off NLR value of 2.5 and the $t$-test was used to compare the continuous variables between those with a low NLR and those with a high NLR. Chi-square test was used to compare the categorical variables between these two groups. Lastly, we performed logistic regression analysis to determine the potential predictors for pathological outcomes in patients with bladder cancer. An initial assessment of predictors was performed using univariate analysis, and only those variables with significance or clinical implications were included in the multivariate analysis. In order to avoid multi-colinearity of continuous and binary NLR, only NLR as a continuous variable was retained in the multivariate analysis. Statistical analysis was performed using Stata 14 for windows (StataCorp, College Station, TX, USA). All tests were two-sided and a value of $p<0.05$ was considered statistically significant.

\section{Results}

Patient characteristics. Three hundred and two patients with bladder cancer were enrolled in our analysis from March 2009 through December 2016, including 144 (47.68\%) with lowgrade disease and $158(52.32 \%)$ with high-grade disease. Patients' clinicopathological characteristics stratified by pathological tumor grading are shown in Table I. The median age was 66 years, and patients with a high-grade disease were significantly older than those with low-grade disease (69 vs. 64 years, $p<0.01)$. There were $66(21.85 \%)$ female patients and $236(78.15 \%)$ male patients; there was no significant difference in gender distribution between those with low-grade disease and those with high-grade disease $(p=0.481)$.

Pathological results suggested most patients had pTa $(n=168)$ or pT1 disease $(n=102)$, accounting for $55.63 \%$ and $33.77 \%$ respectively. There was no significant difference in pathological T-stage distribution between those with low-grade disease and those with high grade disease $(p=0.23)$. All the patients with lymph node metastasis ( $\mathrm{pN} 1, \mathrm{pN} 2)$ had high-grade disease. Median WBC count was $7.28 \times 10^{9} / 1$, neutrophil count was $5.21 \times 10^{9} / 1$, lymphocyte count was $1.43 \times 10^{9} / 1$ and median NLR was 3.83. No significant difference was detected in WBC count in a comparison of patients with low-grade disease and those with high-grade disease. However, those with high-grade disease had a significantly higher neutrophil count $(p=0.003)$, significantly lower lymphocyte count $\left(1.30 \times 10^{9} / 1 \quad v s\right.$. $\left.1.53 \times 10^{9} / 1, p<0.001\right)$ and significantly higher NLR value (4.42 $v s .3 .42, p<0.001$ ) compared to patients with low-grade disease. There were significantly more patients with a high NLR in the high-grade disease group $(58.59 \%)$, and more patients with a low NLR in the low-grade disease group $(66.67 \%)(p<0.001)$. Patients with high-grade disease had a significantly longer hospitalization time (9.5 days $v s .9$ days, $p=0.017$ ).

Pathological outcomes according to NLR. The pathological outcomes according to the NLR group are shown in Table II. More patients had a NLR higher than $2.5(75.17 \%)$.
Within those with low-grade disease, 50 (34.72\%) patients had low NLR and 94 (65.28\%) patients had higher NLR. For high-grade diseases, 25 (15.82\%) patients had lower NLR and $133(84.18 \%)$ patients had a higher NLR. There were significantly more high-grade disease patients in the high NLR patient cohort $(p<0.001)$. There was no significant difference in the distribution of pathological Tstage $(p=0.311)$, muscle invasive bladder cancer $(p=0.841)$ or pathological N-stage $(p=0.181)$ by NLR.

Predictors of pathological high-grade bladder cancer. Univariate and multivariate logistic regression analysis for predictors associated with bladder cancer pathological tumor grading are presented in Table III. From univariate analyses, age $(p<0.001)$, neutrophil count $(p=0.004)$, NLR both as a continuous $(p<0.001)$ and binary $(p<0.001)$ variable were significantly associated with high-grade disease. Multivariate analyses showed that only age $(p<0.001)$ and NLR as a continuous variable $(p=0.001)$ were statistically significantly associated with tumor grade.

\section{Discussion}

More and more studies have found an intimate connection between inflammation and cancer $(1,3,4)$. The hypothesis for this association was based on inflammatory mediators and cytokines released in inflammatory reactions leading to cell damage and gene mutation. These factors are considered to be important elements for tumorigenesis and also help create a microenvironment promoting cancer cell proliferation and metastasis $(10,11)$. Alongside this, inflammation may also accelerate cancer progression by increasing vascular permeability, and up-regulating lymphatic infiltration and stromal invasion at metastatic sites (2). The NLR increases during inflammatory reactions in which the neutrophil granulocyte count increases while the lymphocyte count decreases and thus it could be an effective predictor in cancer. Compared to other assays, the NLR can easily be obtained from peripheral blood tests before treatment, providing a cheap and convenient measure.

Use of the NLR has been proven to be effective in gastrointestinal cancer (3), and a high NLR might also be a prognostic factor in patients with bladder cancer, indicating a worse oncological outcome, thus providing appropriate prognostic information for these patients (12-16). However, the link between elevated NLR and poor survival was unclear, as was the effect of pathological differences on NLR. Hence we conducted this study to compare the pathological outcomes in bladder cancer according to preoperative NLR and to examine whether NLR is predictive of pathological characteristics of the disease.

Our study found that compared to patients with low-grade bladder cancer, those with high-grade disease had a higher 
Table I. Clinicopathological characteristics stratified by pathological tumor grading.

\begin{tabular}{|c|c|c|c|c|}
\hline \multirow[t]{2}{*}{ Characteristic } & \multirow[t]{2}{*}{ Total } & \multicolumn{2}{|c|}{ Pathological tumor grade } & \multirow[t]{2}{*}{ p-Value } \\
\hline & & Low & High & \\
\hline Patients, n (\%) & $302(100)$ & $144(47.68)$ & $158(52.32)$ & \\
\hline Median age (IQR), years & $66(59-75)$ & $64(56-71)$ & $69(61-77)$ & $<0.001$ \\
\hline Gender, n $(\%)$ & & & & 0.481 \\
\hline Female & $66(21.85)$ & $34(51.52)$ & $32(48.48)$ & \\
\hline Male & $236(78.15)$ & $110(46.61)$ & $126(53.39)$ & \\
\hline T-Stage, n (\%) & & & & 0.230 \\
\hline pTis & $2(0.66)$ & $1(50)$ & $1(50)$ & \\
\hline pTa & $168(55.63)$ & $90(53.57)$ & $78(46.43)$ & \\
\hline pT1 & $102(33.77)$ & $39(38.24)$ & $63(61.67)$ & \\
\hline pT2 & $24(7.95)$ & $12(50)$ & $12(50)$ & \\
\hline pT3 & $4(1.32)$ & $1(25)$ & $3(75)$ & \\
\hline pT4 & $2(0.66)$ & $1(50)$ & $1(50)$ & \\
\hline N-Stage, n (\%) & & & & 0.009 \\
\hline $\mathrm{pNO}$ & $292(96.69)$ & $144(49.32)$ & $148(50.68)$ & \\
\hline $\mathrm{pN} 1$ & $4(1.32)$ & $0(0)$ & $4(100)$ & \\
\hline $\mathrm{pN} 2$ & $6(1.99)$ & $0(0)$ & $6(100)$ & \\
\hline Median WBC (IQR), $\times 10^{9} / 1$ & $7.28(6.21-9.16)$ & $7.08(6.06-8.93)$ & $7.52(6.32-9.24)$ & 0.054 \\
\hline Median neutrophils (IQR), $\times 10^{9} / 1$ & $5.21(4.18-7.05)$ & $4.93(3.92-6.76)$ & $5.57(4.44-7.15)$ & 0.003 \\
\hline Median lymphocytes (IQR), $\times 10^{9} / 1$ & $1.43(1.09-1.8)$ & $1.53(1.16-1.90)$ & $1.30(1.04-1.64)$ & $<0.001$ \\
\hline Median NLR (IQR) & $3.83(2.50-5.56)$ & $3.42(2.16-4.91)$ & $4.42(3.00-6.31)$ & $<0.001$ \\
\hline NLR, n (\%) & & & & $<0.001$ \\
\hline$\leq 2.5$ & $75(24.83)$ & $50(66.67)$ & $25(33.33)$ & \\
\hline$>2.5$ & $227(75.17)$ & $94(41.41)$ & $133(58.59)$ & \\
\hline Median hospitalization (IQR), days & $9(6-15)$ & $9(6-14)$ & $9.5(6-18)$ & 0.017 \\
\hline
\end{tabular}

IQR, Interquartile range; NLR, neutrophil-to-lymphocyte ratio; WBC, white blood cells.

neutrophil count and lower lymphocyte count, and accordingly a higher NLR. Defining 2.5 as a NLR cut-off value, compared with patients with a low NLR, those with high NLR had high-grade disease. Regression analysis suggested NLR was a predictor of high-grade disease, while the WBC count failed to predict high-grade disease. This might be the reason why high NLR groups had worse survival in other studies $(7,14,17)$.

In a recent study, Celik et al. found that the NLR might act as a significant biomarker in the staging of bladder cancer (12). They analyzed 222 patients with bladder cancer, including 59 cases with low-grade disease and 103 with high-grade, and found the NLR between these two groups was significantly different. However, in our study, we did not find the difference in NLR value according to pathological staging (non-muscleinvasive $v s$. muscle-invasive), instead, we found a significant difference in NLR according to the pathological grading of bladder cancer. This means the value of NLR is yet to be clarified. Larger scale and well-designed studies are required before NLR becomes an effective biomarker in the clinic.

The limitation of this study includes its retrospective design and limited sample size from a single institution. In
Table II. Pathological outcomes according to the neutrophil-tolymphocyte ratio (NLR).

\begin{tabular}{lccc}
\hline Characteristic & \multicolumn{2}{c}{ NLR, n (\%) } & \multirow{2}{*}{-Value } \\
\cline { 2 - 3 } & $\leq 2.5$ & $>2.5$ & \\
\hline Total patients & $75(24.83)$ & $227(75.17)$ & \\
Pathological tumor grade & & & $<0.001$ \\
Low & $50(34.72)$ & $94(65.28)$ & \\
High & $25(15.82)$ & $133(84.18)$ & \multirow{2}{*}{0.311} \\
T-Stage & $1(50)$ & $1(50)$ & \\
pTis & $47(27.98)$ & $121(72.02)$ & \\
pTa & $20(19.61)$ & $82(80.39)$ & \\
pT1 & $4(16.67)$ & $20(83.33)$ & \\
pT2 & $2(50)$ & $2(50)$ & \\
pT3 & $1(50)$ & $1(50)$ & \\
pT4 & & & 0.841 \\
Muscle invasion & $68(25.00)$ & $204(75.00)$ & \\
No (pTis, pTa, pT1) & $7(23.33)$ & $23(76.67)$ & \multirow{2}{*}{0.181} \\
Yes (pT2, pT3, pT4) & & & \\
N-Stage & $75(25.68)$ & $217(74.32)$ & \\
pN0 & $0(0)$ & $4(100)$ & \\
pN1 & $0(0)$ & $6(100)$ & \\
pN2 & & & \\
\hline
\end{tabular}


Table III. Predictors of pathological tumor grading by logistic regression analysis.

\begin{tabular}{|c|c|c|c|c|c|c|c|c|}
\hline \multirow[t]{2}{*}{ Variable } & \multicolumn{2}{|c|}{ Pathological tumor grade } & \multicolumn{3}{|c|}{ Univariate } & \multicolumn{3}{|c|}{ Multivariate } \\
\hline & Low & High & OR & $95 \% \mathrm{CI}$ & $p$-Value & OR & $95 \% \mathrm{CI}$ & $p$-Value \\
\hline Median age (IQR), years & $64(56-71)$ & $69(61-77)$ & 1.04 & $1.02-1.06$ & $<0.001$ & 1.04 & $1.02-1.06$ & $<0.001$ \\
\hline Gender, n (\%) & & & 1.22 & $0.70-2.10$ & 0.481 & 1.21 & $0.67-2.18$ & 0.519 \\
\hline Female & $34(51.52)$ & $32(48.48)$ & & & & & & \\
\hline Male & $110(46.61)$ & $126(53.39)$ & & & & & & \\
\hline T-Stage, n (\%) & & & 1.24 & $0.93-1.64$ & 0.137 & 1.16 & $0.86-1.56$ & 0.344 \\
\hline pTis & $1(50)$ & $1(50)$ & & & & & & \\
\hline pTa & $90(53.57)$ & $78(46.43)$ & & & & & & \\
\hline pT1 & $39(38.24)$ & $63(61.67)$ & & & & & & \\
\hline pT2 & $12(50)$ & $12(50)$ & & & & & & \\
\hline pT3 & $1(25)$ & $3(75)$ & & & & & & \\
\hline pT4 & $1(50)$ & $1(50)$ & & & & & & \\
\hline Muscle invasion, $\mathrm{n}(\%)$ & & & 1.05 & $0.49-2.23$ & 0.907 & & & \\
\hline No (pTis, pTa, pT1) & $130(47.79)$ & $142(52.21)$ & & & & & & \\
\hline Yes (pT2, pT3, pT4) & $14(46.67)$ & $16(53.33)$ & & & & & & \\
\hline Median WBC (IQR), $\times 10^{9} / 1$ & $7.08(6.06-8.93)$ & $7.52(6.32-9.24)$ & 1.11 & $1.00-1.23$ & 0.057 & 0.98 & $0.85-1.13$ & 0.776 \\
\hline Median neutrophils (IQR), $\times 10^{9} / 1$ & $4.93(3.92-6.76)$ & $5.57(4.44-7.15)$ & 1.19 & $1.06-1.33$ & 0.004 & & & \\
\hline Median NLR (IQR) & $3.42(2.16-4.91)$ & $4.42(3.00-6.31)$ & 1.29 & $1.15-1.44$ & $<0.001$ & 1.28 & $1.11-1.47$ & 0.001 \\
\hline NLR, n (\%) & & & 2.83 & $1.64-4.89$ & $<0.001$ & & & \\
\hline$\leq 2.5$ & $50(66.67)$ & $25(33.33)$ & & & & & & \\
\hline$>2.5$ & $94(41.41)$ & $133(58.59)$ & & & & & & \\
\hline
\end{tabular}

95\% CI, 95\% Confidence interval; IQR, interquartile range; OR, odds ratio; WBC, white blood cells.

addition, we did not obtain the survival data for these patients and thus cannot establish a link of NLR with survival.

In conclusion, our study indicated that preoperative NLR was associated with pathological tumor grade, but not associated with pathological tumor staging in patients with bladder cancer. A high preoperative NLR could be helpful in predicting the pathological grade of bladder cancer.

\section{References}

1 Herszényi L, Lakatos G, Hritz I, Varga MZ, Cierny G and Tulassay Z: The role of inflammation and proteinases in tumor progression. Dig Dis Basel Switz 30: 249-254, 2012.

2 Formica V, Luccchetti J, Cunningham D, Smyth EC, Ferroni P, Nardecchia A, Tesauro M, Cereda V, Guadagni F and Roselli M: Systemic inflammation, as measured by the neutrophil/ lymphocyte ratio, may have differential prognostic impact before and during treatment with fluorouracil, irinotecan and bevacizumab in metastatic colorectal cancer patients. Med Oncol 31: 166, 2014.

3 Jaramillo-Reta KY, Velázquez-Dohorn ME and Medina-Franco $\mathrm{H}$ : Neutrophil to lymphocyte ratio as predictor of surgical mortality and survival in complex surgery of the upper gastrointestinal tract. Rev Investig Clin Organo Hosp Enfermedades Nutr 67: 117-121, 2015.

4 Cho H, Hur HW, Kim SW, Kim SH, Kim JH, Kim YT and Lee $\mathrm{K}$ : Pre-treatment neutrophil to lymphocyte ratio is elevated in epithelial ovarian cancer and predicts survival after treatment. Cancer Immunol Immunother 58: 15-23, 2009.
5 Mano R, Baniel J, Shoshany O, Margel D, Bar-On T, Nativ O, Rubinstein $\mathrm{J}$ and Halachmi S: Neutrophil-to-lymphocyte ratio predicts progression and recurrence of non-muscle-invasive bladder cancer. Urol Oncol 33: 67.e1-7, 2015.

6 Krane LS, Richards KA, Kader AK, Davis R, Balaji KC and Hemal AK: Preoperative neutrophil/lymphocyte ratio predicts overall survival and extravesical disease in patients undergoing radical cystectomy. J Endourol Endourol Soc 27: 1046-1050, 2013.

7 Taguchi S, Nakagawa T, Matsumoto A, Nagase Y, Kawai T, Tanaka Y, Yoshida K, Yamamoto S, Enomoto Y, Nose Y, Sato T, Ishikawa A, Uemura Y, Fujimura T, Fukuhara H, Kume H and Homma Y: Pretreatment neutrophil-to-lymphocyte ratio as an independent predictor of survival in patients with metastatic urothelial carcinoma: A multi-institutional study. Int J Urol Off J Jpn Urol Assoc 22: 638-643, 2015.

8 Bhindi B, Hermanns T, Wei Y, Yu J, Richard PO, Wettstein MS, Templeton A, Li K, Sridhar SS, Jewett MAS, Fleshner NE, Zlotta AR and Kulkarni GS: Identification of the best complete blood count-based predictors for bladder cancer outcomes in patients undergoing radical cystectomy. Br J Cancer 114: 207212, 2016.

9 Edge SB and Compton CC: The American Joint Committee on Cancer: the 7th edition of the AJCC cancer staging manual and the future of TNM. Ann Surg Oncol 17: 1471-1474, 2010.

10 Hanahan D and Weinberg RA: Hallmarks of cancer: the next generation. Cell 144: 646-674, 2011.

11 Grivennikov SI, Greten FR and Karin M: Immunity, inflammation, and cancer. Cell 140: 883-899, 2010.

12 Celik O, Akand M, Keskin MZ, Yoldas M and Ilbey YO: Preoperative neutrophil-to-lymphocyte ratio (NLR) may be 
predictive of pathologic stage in patients with bladder cancer larger than $3 \mathrm{~cm}$. Eur Rev Med Pharmacol Sci 20: 652-656, 2016.

13 Bhindi B, Hermanns T, Wei Y, Yu J, Templeton A, Noon A, Bhatt J, Richard P, Jewett MAS, Hamilton R, Finelli A, Fleshner N, Zlotta A and Kulkarni G: Determining the best complete blood count-based biomarker for oncologic outcomes following radical cystectomy for urothelial carcinoma of the bladder. J Urol 193: e856, 2015.

14 Buisan O, Orsola A, Areal J, Font A, Oliveira M, Martinez R and Ibarz L: Low pretreatment neutrophil-to-lymphocyte ratio predicts for good outcomes in patients receiving neoadjuvant chemotherapy before radical cystectomy for muscle invasive bladder cancer. Clin Genitourin Cancer 15: 145-151.e2, 2017

15 Mbeutcha A, Shariat SF, Rieken M, Rink M, Xylinas E, Seitz C, Lucca I, Mathieu R, Rouprêt M, Briganti A, Karakiewicz PI and Klatte T: Prognostic significance of markers of systemic inflammatory response in patients with non-muscle-invasive bladder cancer. Urol Oncol 34: 483.e17-483.e24, 2016.
16 van Kessel KEM, de Haan LM, Fransen van de Putte EE, van Rhijn BWG, de Wit R, van der Heijden MS, Zwarthoff EC and Boormans JL: Elevated derived neutrophil-to-lymphocyte ratio corresponds with poor outcome in patients undergoing preoperative chemotherapy in muscle-invasive bladder cancer. Bladder Cancer Amst Neth 2: 351-360, 2016.

17 Gondo T, Nakashima J, Ohno Y, Choichiro O, Horiguchi Y, Namiki K, Yoshioka K, Ohori M, Hatano T and Tachibana M: Prognostic value of neutrophil-to-lymphocyte ratio and establishment of novel preoperative risk stratification model in bladder cancer patients treated with radical cystectomy. Urology 79: 1085-1091, 2012.

Received May 2, 2017

Revised June 7, 2017

Accepted June 13, 2017 